\title{
STRUCTURAL PROPERTIES OF RECURSIVELY PARTITIONABLE GRAPHS WITH CONNECTIVITY 2
}

\author{
Olivier BAudon \\ Université Bordeaux, LaBRI, UMR 5800, F-33400 Talence, France \\ CNRS, LaBRI, UMR 5800, F-33400 Talence, France \\ e-mail: olivier.baudon@labri.fr \\ Julien Bensmail \\ Department of Applied Mathematics and Computer Science \\ Technical University of Denmark \\ DK-2800 Lyngby, Denmark \\ e-mail: julien.bensmail.phd@gmail.com \\ Florent FoucAud \\ LIMOS - CNRS UMR 6158 \\ Université Blaise Pascal \\ Clermont-Ferrand, France
}

e-mail: florent.foucaud@gmail.com

AND

MONIKA PILŚNIAK

Department of Discrete Mathematics

AGH University of Science and Technology

A. Mickiewicza 30, 30-059 Kraków, Poland

e-mail: pilsniak@agh.edu.pl

\begin{abstract}
A connected graph $G$ is said to be arbitrarily partitionable (AP for short) if for every partition $\left(n_{1}, \ldots, n_{p}\right)$ of $|V(G)|$ there exists a partition $\left(V_{1}, \ldots, V_{p}\right)$ of $V(G)$ such that each $V_{i}$ induces a connected subgraph of $G$ on $n_{i}$ vertices. Some stronger versions of this property were introduced, namely the ones of being online arbitrarily partitionable and recursively arbitrarily partitionable (OL-AP and R-AP for short, respectively), in which
\end{abstract}


the subgraphs induced by a partition of $G$ must not only be connected but also fulfil additional conditions. In this paper, we point out some structural properties of OL-AP and R-AP graphs with connectivity 2. In particular, we show that deleting a cut pair of these graphs results in a graph with a bounded number of components, some of whom have a small number of vertices. We obtain these results by studying a simple class of 2 -connected graphs called balloons.

Keywords: online arbitrarily partitionable graph, recursively arbitrarily partitionable graph, graph with connectivity 2 , balloon graph.

2010 Mathematics Subject Classification: 68R10, 05C75.

\section{REFERENCES}

[1] D. Barth, O. Baudon and J. Puech, Decomposable trees: a polynomial algorithm for tripodes, Discrete Appl. Math. 119 (2002) 205-216. doi:10.1016/S0166-218X(00)00322-X

[2] D. Barth and H. Fournier, A degree bound on decomposable trees, Discrete Math. 306 (2006) 469-477. doi:10.1016/j.disc.2006.01.006

[3] O. Baudon, F. Foucaud, J. Przybyło and M. Woźniak, On the structure of arbitrarily partitionable graphs with given connectivity, Discrete Appl. Math. 162 (2014) 381-385.

doi:10.1016/j.dam.2013.09.007

[4] O. Baudon, F. Gilbert and M. Woźniak, Recursively arbitrarily vertex-decomposable suns, Opuscula Math. 31 (2011) 533-547. doi:10.7494/OpMath.2011.31.4.533

[5] O. Baudon, F. Gilbert and M. Woźniak, Recursively arbitrarily vertex-decomposable graphs, Opuscula Math. 32 (2012) 689-706. doi:10.7494/OpMath.2012.32.4.689

[6] J. Bensmail, On the longest path in a recursively partitionable graph, Opuscula Math. 33 (2013) 631-640. doi:10.7494/OpMath.2013.33.4.631

[7] S. Cichacz, A. Görlich, A. Marczyk, J. Przybyło and M. Woźniak, Arbitrarily vertex decomposable caterpillars with four or five leaves, Discuss. Math. Graph Theory 26 (2006) 291-305.

doi:10.7151/dmgt.1321

[8] R. Diestel, Graph Theory (Springer, Berlin Heidelberg, 2005).

[9] E. Győri, On division of graphs to connected subgraphs, in: Combinatorics, Proc. Fifth Hungariam Colloq., Keszthely, 1976, Vol. I, Colloq. Math. Soc. János Bolyai 18 (1978) 485-494. 
[10] M. Horňák, Zs. Tuza and M. Woźniak, On-line arbitrarily vertex decomposable trees, Discrete Appl. Math. 155 (2007) 1420-1429.

doi:10.1016/j.dam.2007.02.011

[11] M. Horňák and M. Woźniak, Arbitrarily vertex decomposable trees are of maximum degree at most six, Opuscula Math. 23 (2003) 49-62.

[12] L. Lovász, A homology theory for spanning trees of a graph, Acta Math. Hungar. 30 (1977) 241-251.

[13] A. Marczyk, An Ore-type condition for arbitrarily vertex decomposable graphs, Discrete Math. 309 (2009) 3588-3594. doi:10.1016/j.disc.2007.12.066

Received 9 October 2014

Revised 25 February 2015

Accepted 25 February 2015

\section{A. Details for the Proof of Lemma ??}

Let $B=B\left(b_{1}, \overline{b_{2}}, \overline{b_{3}}, \underline{b_{4}}, \underline{b_{5}}\right)$, where $b_{2} \leq b_{3}$, and $b_{2} \leq b_{4} \leq b_{5}$. In case $b_{2}=b_{4}$, we assume $b_{3} \leq b_{5}$. We end the proof of Lemma ?? by showing that, no matter what are $b_{1}, b_{2}, b_{3}, b_{4}, b_{5}$, there is always a $\lambda \in\{1, \ldots, n-1\}$ such that, for every partition $(S, T)$ of $V\left(B^{+}\right)$with $|S|=\lambda$ and $B^{+}[S], B^{+}[T]$ being connected, one of the following conditions is necessarily met.

1. $B^{+}[T] \in \mathcal{B}_{2}^{2}(1)$ - hence $B^{+}[T]$ is not OL-AP by the induction hypothesis;

2. $B^{+}[T] \in \mathcal{B}_{1}^{2}(1) \cup \mathcal{B}_{2}^{1}(1)$, and $B[T]$ is a tree not listed in Theorem ?? - hence $B^{+}[T]$ is not OL-AP by Lemma ??;

3. $r_{1}, r_{2} \in T$, and $B^{+}[T]$ is a tree with $\Delta\left(B^{+}[T]\right)=4$, or in which both $r_{1}$ and $r_{2}$ have degree at least 3 - hence not OL-AP according to Theorem ??.

We basically proceed by case analysis, that is consider the possible values of $b_{1}, b_{2}, b_{3}, b_{4}, b_{5}$. For every case, we indicate below the appropriate choices of $\lambda$ only, as well as, when applicable, the sets of graphs in $\mathcal{B}_{1}^{2}(1) \cup \mathcal{B}_{2}^{1}(1)$ (second situation above) which appear as $B^{+}[T]$ for the indicated values of $\lambda$.

(1) $b_{2} \geq 2: \lambda=1$.

(2) $b_{2}=1$.

(2.1) $b_{3}=1$.

(2.1.1) $b_{4} \geq 3: \lambda=2$.

(2.1.2) $b_{4}=2$.

(2.1.2.1) $b_{5} \in\{4,6\}$.

(2.1.2.1.1) $b_{1}=8-b_{5}: \lambda=3 \rightarrow P\left(2,8-b_{5}, b_{5}\right) \in\{P(2,4,4), P(2,2,6)\}$. 
(2.1.2.1.2) $b_{1} \neq 8-b_{5}: \lambda=1 \rightarrow P\left(2, b_{1}+2, b_{5}\right) \neq P(2,4,6)$.

(2.1.2.2) $b_{5} \notin\{4,6\}: \lambda=1 \rightarrow P\left(2, b_{1}+2, b_{5}\right) \neq P(2,4,6)$.

(2.1.3) $b_{4}=1$.

(2.1.3.1) $b_{5} \neq 2: \lambda=2$.

(2.1.3.2) $b_{5}=2$.

(2.1.3.2.1) $b_{1} \equiv 1(\bmod 2): \lambda=2 \rightarrow \operatorname{Cat}\left(2, b_{1}+3\right)$.

(2.1.3.2.2) $b_{1} \equiv 0(\bmod 2)$.

(2.1.3.2.2.1) $b_{1}=6 p+2: \lambda=3 \rightarrow \operatorname{Cat}(3,6 p+3)$.

(2.1.3.2.2.2) $b_{1}=6 p+4: \lambda=5 \rightarrow \operatorname{Cat}(2,6 p+4), \operatorname{Cat}(3,6 p+3)$.

(2.1.3.2.2.3) $b_{1}=6 p+6: \lambda=7 \rightarrow \operatorname{Cat}(2,6 p+4), \operatorname{Cat}(3,6 p+3)$.

(2.2) $b_{3}=2$.

(2.2.1) $b_{4}=1$.

(2.2.1.1) $b_{5} \geq 4: \lambda=3$.

(2.2.1.2) $b_{5}=3$.

(2.2.1.2.1) $b_{1} \equiv 1(\bmod 2): \lambda=4 \rightarrow \operatorname{Cat}\left(4, b_{1}+1\right)$.

(2.2.1.2.2) $b_{1} \equiv 0(\bmod 2)$.

(2.2.1.2.2.1) $b_{1}=6 p+2: \lambda=5 \rightarrow \operatorname{Cat}(3,6 p+3), \operatorname{Cat}(4,6 p+2)$.

(2.2.1.2.2.2) $b_{1}=6 p+4: \lambda=7 \rightarrow \operatorname{Cat}(3,6 p+3)$, Cat $(4,6 p+2)$.

(2.2.1.2.2.3) $b_{1}=6 p+6: \lambda=3 \rightarrow \operatorname{Cat}(3,6 p+9)$.

(2.2.1.3) $b_{5}=2: \lambda=3$.

(2.2.2) $b_{4}=2$.

(2.2.2.1) $b_{5} \geq 4: \lambda=3$.

(2.2.2.2) $b_{5}=3$.

(2.2.2.2.1) $b_{1} \geq 2: \lambda=4 \rightarrow P\left(2,3, b_{1}\right)$.

(2.2.2.2.2) $b_{1}=1: \lambda=2 \rightarrow P(2,3,3)$, Cat $(3,6)$.

(2.2.2.3) $b_{5}=2: \lambda=3$.

(2.2.3) $b_{4} \geq 3: \lambda=1 \rightarrow P\left(b_{4}, b_{5}, b_{1}+3\right)$ with $\min \left\{b_{4}, b_{5}, b_{1}+3\right\} \geq 3$.

(2.3) $b_{3} \geq 3$.

(2.3.1) $b_{4}=1: \lambda=2$.

(2.3.2) $b_{4}=2$.

(2.3.2.1) $b_{5} \geq 4$.

(2.3.2.1.1) $b_{3} \geq 4: \lambda=3$.

(2.3.2.1.2) $b_{3}=3$. 
(2.3.2.1.2.1) $b_{5} \geq 5: \lambda=4$.

$(2.3 .2 .1 .2 .2) b_{5}=4$.

(2.3.2.1.2.2.1) $b_{1}=2: \lambda=2 \rightarrow \operatorname{Cat}(4,8)$.

(2.3.2.1.2.2.2) $b_{1} \neq 2: \lambda=1 \rightarrow P\left(2,4, b_{1}+4\right)$.

(2.3.2.2) $b_{5} \in\{2,3\}: \lambda=1 \rightarrow P\left(2, b_{1}+4, b_{5}\right)$.

(2.3.3) $b_{4} \geq 3: \lambda=1 \rightarrow P\left(b_{1}+1+b_{3}, b_{4}, b_{5}\right)$.

\section{B. Proofs of Lemmas ?? to ??}

This appendix gathers all the proofs of Lemmas ?? to ?? of Section ??, as well as some intermediate lemmas needed to prove these statements. It is worth mentioning that these proofs often make implicit use of the full characterization of OL-AP trees (Theorem ??) and the two sufficient conditions for a graph to be OL-AP given by Observations ?? and ??. In all these proofs, it is assumed that $x \leq y$ or $x \leq y \leq z$ generally holds when the corresponding elements have been introduced. Given a graph $G$ and an integer $\lambda \in\{1, \ldots, n-1\}$, an OLAP-partition of $G$ for $\lambda$ is a partition $(S, T)$ of $V$ such that $G[S]$ and $G[T]$ are connected on $\lambda$ vertices and OL-AP on $n-\lambda$ vertices, respectively. According to Definition ??, the graph $G$ is OL-AP if and only if either $G$ is an isolated vertex or $G$ admits an OL-AP-partition for every $\lambda \in\{1, \ldots, n-1\}$.

Lemma B.1. The graph $B\left(12^{+}, 12^{+}, \bar{x}, \bar{y}\right)$ is not OL-AP for every $x \geq 1$ and $y \geq 10$.

Proof. Let us prove this claim by induction on $x+y$ as we did to prove Lemma ?? As a base case, let us consider the graph $B=B\left(12^{+}, 12^{+}, \overline{1}, \overline{10}\right)$. Observe that $B$ is not OL-AP since it does not admit an OL-AP-partition for 11. Indeed, every possible choice of 11 vertices as $S$ inducing a connected subgraph of $B$ makes $B[T]$ being either disconnected, a caterpillar Cat $\left(11,15^{+}\right)$, or a tree with maximum degree 4 . For similar reasons, observe that neither $B\left(12^{+}, 12^{+}, \overline{1}, \overline{11}\right)$ nor $B\left(12^{+}, 12^{+}, \overline{2}, \overline{10}\right)$ are OL-AP since they do not admit an OL-AP-partition for 12 and 11 , respectively.

Let us now suppose that this lemma holds whenever $x+y \leq k-1$ for some $k \geq 13$, and consider a graph $B=B\left(12^{+}, 12^{+}, \bar{x}, \bar{y}\right)$ such that $x+y=k$. We claim that there exists a $\lambda \in\{1, \ldots, n-1\}$ such that $B$ does not admit an OL-AP-partition for $\lambda$, and thus that $B$ is not OL-AP.

- $x>1$ and $y>10$ : under these conditions, there does not exist an OL-APpartition of $B$ for 1. Indeed, every possible choice for $S$ which does not make $B[T]$ being disconnected makes this subgraph being isomorphic to either a non-caterpillar 3-pode different from $P(2,4,6)$, a tree with maximum degree 4 , or a graph not OL-AP according to the induction hypothesis. 
- $x=1$ and $y>11$ : observe that there does not exist an OL-AP-partition of $B$ for 2 , since every coherent choice for $S$ makes $B[T]$ being disconnected or isomorphic to either a caterpillar $\operatorname{Cat}\left(13^{+}, 13^{+}\right)$, a tree with maximum degree 4 , or a partial balloon which is not OL-AP by the induction hypothesis.

- $x>2$ and $y=10$ : once again, $B$ does not admit an OL-AP-partition for 11 since every choice of 11 vertices as $S$ inducing a connected subgraph of $B$ makes $B[T]$ being either disconnected, a tree with maximum degree 4 , or a non-OL-AP 3-pode.

Lemma B.2. The graph $B\left(12^{+}, 12^{+}, \bar{x}, \bar{y}, \underline{z}\right)$ is not OL-AP for every $x, y, z \geq 1$.

Proof. We prove this claim by induction on $x+y+z$. Let us first suppose that $x=y=z=1$ and consider the associated graph $B=B\left(12^{+}, 12^{+}, \overline{1}, \overline{1}, \underline{1}\right)$. Once again, $B$ is not OL-AP since there does not exist an OL-AP-partition of $B$ for 2. Indeed, every possible choice for $S$ makes $B[T]$ being either disconnected, or isomorphic to either a tree with maximum degree 4 or a tree having two degree-3 vertices.

To complete the base case, observe that $B\left(12^{+}, 12^{+}, \overline{1}, \overline{2}, \underline{1}\right)$ and $B\left(12^{+}, 12^{+}\right.$, $\overline{1}, \overline{1}, 2)$ are not OL-AP since they do not admit an OL-AP-partition for 3 . Indeed, for every coherent choice of $S$, the subgraph $B[T]$ is disconnected, or isomorphic to either a tree with maximum degree 4 , a tree having two degree- 3 vertices, or a non-caterpillar 3-pode different from $P(2,4,6)$.

Suppose now that this claim holds whenever $x+y+z \leq k-1$ for some $k \geq 5$, and consider a balloon $B=B\left(12^{+}, 12^{+}, \bar{x}, \bar{y}, \underline{z}\right)$ where $x+y+z=k$. Once again, we consider two main cases.

- $z>1$ : in this case, $B$ is not OL-AP since it cannot be OL-AP-partitioned for 1. Indeed, observe that removing one vertex from $B$ makes the remaining subgraph being disconnected, isomorphic to a tree with maximum degree 4 or two degree-3 vertices, or isomorphic to a partial balloon which is not OL-AP according to the induction hypothesis or Lemma ??.

- $z=1$ : once again, $B$ is not OL-AP under this condition since it cannot be OL-AP-partitioned for 2. Indeed, for every coherent choice as $S$, the remaining graph $B[T]$ is indeed not connected, a tree with maximum degree 4 or two degree- 3 vertices, or a partial balloon which is not OL-AP according to our induction hypothesis or previous Lemma??.

Proof of Lemma ??. Once more, let us prove this claim by induction on $x+y$. Consider first that $x=y=1$ and let $B=B\left(12^{+}, 12^{+}, 12^{+}, \overline{1}, \underline{1}\right)$. Observe that $B$ is not OL-AP because it cannot be OL-AP-partitioned for 2 . Indeed, every possible choice for $S$ makes $B[T]$ being disconnected, isomorphic to a tree with maximum degree 4 , to a partial balloon which is not OL-AP by Lemma B.2, or to a partial 6-balloon. In the latter case, such a graph cannot be OL-AP since otherwise there would exist an OL-AP 6-balloon contradicting Theorem ??. 
Additionally, observe that $B=B\left(12^{+}, 12^{+}, 12^{+}, \overline{1}, \underline{2}\right)$ cannot be OL-APpartitioned for 3. Indeed, for every coherent choice for $S$, the subgraph $B[T]$ is not OL-AP for the same reasons as in the previous case. Hence, $B$ is not OL-AP.

We now suppose that this claim holds for every $x+y \leq k-1$ for some $k \geq 4$, and consider a partial balloon $B=B\left(12^{+}, 12^{+}, 12^{+}, \bar{x}, \underline{y}\right)$ where $x+y=k$. Let us take the following two cases in consideration to show that $B$ is not OL-AP.

- $x>1$ and $y>1$ : notice that, in this situation, $B$ cannot be OL-APpartitioned for 1 . Indeed, for some similar reasons as the ones we used for the base cases, we have to consider $S=\left\{v_{1}^{4}\right\}$ or $S=\left\{v_{1}^{5}\right\}$. But in both cases, $B[T]$ cannot be OL-AP by the induction hypothesis.

- $x=1$ and $y>2$ : once again, observe that $B$ cannot be OL-AP-partitioned for 2. Indeed, observe that we must consider $S=\left\{v_{1}^{5}, v_{2}^{5}\right\}$ since otherwise there would exist an OL-AP 6-balloon, an OL-AP tree having maximum degree 4, or a graph contradicting Lemma B.2. But for this choice of $S$, we have $B[T]=B\left(12^{+}, 12^{+}, 12^{+}, \overline{1}, \underline{y-2}\right)$ which is not OL-AP according to our induction hypothesis.

Proof of Lemma ??. Once again, this claim is proved by induction on $x$. Let us first suppose that $x=1$ and let $B$ be the partial balloon $B\left(12^{+}, 12^{+}, 12^{+}, \overline{1}\right)$. This time, $B$ is not OL-AP since it cannot be OL-AP-partitioned for 2 . Indeed, for every possible choice of $S$, the remaining graph $B[T]$ is not OL-AP since it is disconnected, isomorphic to a tree with maximum degree 4 , to a non-caterpillar 3 -pode different from $P(2,4,6)$ or to a partial balloon which is not OL-AP by Lemma ??, B.1 or B.2.

Let us now suppose that this claim holds for every $x \leq k-1$ and some $k \geq 2$. To complete the proof, observe that a graph $B=B\left(12^{+}, 12^{+}, 12^{+}, \bar{k}\right)$ is not OL-AP since it cannot be OL-AP-partitioned for 1 . Indeed, for every choice of $S$, the subgraph $B[T]$ is not OL-AP according to the induction hypothesis, or because of one reason used for the base case.

Lemma B.3. The graph $B\left(12^{+}, 12^{+}, \bar{x}, \bar{y}, \bar{z}\right)$ is not OL-AP for every $x, y, z \geq 1$.

Proof. We prove this claim by induction on $x+y+z$. First, let us suppose that $x=y=z=1$ and consider the partial balloon $B=B\left(12^{+}, 12^{+}, \overline{1}, \overline{1}, \overline{1}\right)$. Notice that $B$ is not OL-AP since it cannot be OL-AP-partitioned for 2 . Indeed, every choice of $S$ implies that $B[T]$ is either disconnected or isomorphic to a tree with maximum degree at least 4 . Analogously, observe that neither $B\left(12^{+}, 12^{+}, \overline{1}, \overline{1}, \overline{2}\right)$ nor $B\left(12^{+}, 12^{+}, \overline{1}, \overline{2}, \overline{2}\right)$ are OL-AP since they cannot be OL-AP-partitioned for 3 , and that $B\left(12^{+}, 12^{+}, \overline{1}, \overline{1}, \overline{3}\right)$ is not OL-AP as it cannot be OL-AP-partitioned for 2 . 
Suppose now that this claim holds by induction whenever $x+y+z \leq k-1$ for a $k \geq 6$, and consider a partial balloon $B=B\left(12^{+}, 12^{+}, \bar{x}, \bar{y}, \bar{z}\right)$ where $x+y+z=k$. We distinguish the following two main cases depending on $x, y$ and $z$.

- $x>1, y>1$ and $z>1$ : suppose we want to OL-AP-partition $B$ for 1 . Then, we must consider $S=\left\{v_{1}^{3}\right\}, S=\left\{v_{1}^{4}\right\}$ or $S=\left\{v_{1}^{5}\right\}$ since, for every other choice of $S$, the remaining graph $B[T]$ is either disconnected or isomorphic to a tree having maximum degree at least 4 . But in any of these three choices for $S$, the subgraph $B[T]$ is not OL-AP by the induction hypothesis. Thus, $B$ is not OL-AP.

- $x=1$ : let $\alpha=\min (\{2,3,4\} \backslash\{y, z\})$. In this situation, $B$ cannot be OLAP for the same reason as above but for an OL-AP-partition of $B$ for $\alpha$. Indeed, for every coherent choice of $S$, the remaining graph $B[T]$ is not OLAP either according to the induction hypothesis, or because it is isomorphic to a non-connected graph or a tree with maximum degree at least 4 .

Lemma B.4. The graph $B\left(12^{+}, 12^{+}, 12^{+}, \bar{x}, \bar{y}\right)$ is not OL-AP for every $x, y \geq 1$.

Proof. Once again, we prove this claim by induction on $x+y$. We first suppose that $x=y=1$ and let $B=B\left(12^{+}, 12^{+}, 12^{+}, \overline{1}, \overline{1}\right)$. Similarly as in the proofs of the previous lemmas, $B$ is not OL-AP because it cannot be OL-AP-partitioned for 2. Indeed, for every possible choice of $S$, the remaining graph $B[T]$ is not connected, a tree with maximum degree 5 , a partial balloon not OL-AP according to Lemma B.2 or B.3, or a partial 6-balloon. For the latter case, recall that a partial 6-balloon cannot be OL-AP since otherwise there would exist a 6 -balloon contradicting Theorem ??. Similarly, observe that $B\left(12^{+}, 12^{+}, 12^{+}, \overline{1}, \overline{2}\right)$ is not OL-AP since it cannot be OL-AP-partitioned for 3.

We finally suppose that the induction hypothesis is true whenever $x+y \leq k-1$ for some $k \geq 4$, and consider a partial balloon $B=B\left(12^{+}, 12^{+}, 12^{+}, \bar{x}, \bar{y}\right)$ where $x+y=k$. We distinguish two main cases, depending on the values of $x$ and $y$, to prove that $B$ is not OL-AP.

- $x>1$ and $y>1$ : in this situation, $B$ is not OL-AP since it cannot be OL-AP-partitioned for 1 . Indeed, for every choice of $S$, the remaining graph $B[T]$ is not OL-AP either for one of the reasons used for the base cases or according to the induction hypothesis.

- $x=1$ and $y>2$ : the above arguments hold to prove that $B$ cannot be OL-AP-partitioned for 2. Thus, $B$ is not OL-AP.

Proof of Lemma ??. Let us prove this claim by induction on $x$. We first suppose that $x=1$ and consider the OL-AP-partition of $B=B\left(12^{+}, 12^{+}, 12^{+}, 12^{+}, \overline{1}\right)$ for 2 . Such a partition does not exist since for every choice of $S$, the remaining graph $B[T]$ cannot be OL-AP. Indeed, this subgraph is either not connected, a tree with maximum degree at least 4 , a partial balloon which cannot be OL-AP 
according to Lemma ?? or B.4, or a partial 6-balloon. In the latter case, such a graph cannot be OL-AP since otherwise there would exist a graph contradicting Theorem ??.

Suppose now that $B\left(12^{+}, 12^{+}, 12^{+}, 12^{+}, \bar{x}\right)$ is not OL-AP for every $x \leq k-1$ and some $k \geq 2$, and consider a graph $B=B\left(12^{+}, 12^{+}, 12^{+}, 12^{+}, \bar{k}\right)$. Once again, $B$ cannot be OL-AP since it cannot be OL-AP-partitioned for 1 . Indeed, for every possible choice for $S$, the graph $B[T]$ cannot be OL-AP either according to the induction hypothesis or because of one of the reasons used for the base case. 